\title{
Biomechanical analysis of acute subdural hematoma resulting from judo
}

\author{
Masahito Hitosugi ${ }^{1}$, Haruo Murayama ${ }^{2}$, Yasuki Motozawa ${ }^{3}$, Kanto IshiI $^{3}$, Masahiro Ogino ${ }^{4}$, and Katsuhiro \\ KOYAMA $^{5}$ \\ ${ }^{1}$ Department of Legal Medicine, Shiga University of Medical Science, Otsu, Shiga, Japan; ${ }^{2}$ Department of Sports Science, Dokkyo \\ Medical University School of Medicine, Shimotsuga, Tochigi, Japan; ${ }^{3}$ Department of Legal Medicine, Dokkyo Medical University \\ School of Medicine, Shimotsuga, Tochigi, Japan; ${ }^{4}$ Department of Neurosurgery, Dokkyo Medical University School of Medicine, \\ Shimotsuga, Tochigi, Japan; and ${ }^{5}$ Department of Health Sciences, Interdisciplinary Graduate School of Medicine and Engineering, Uni- \\ versity of Yamanashi, Kofu, Yamanashi, Japan
}

(Received 21 August 2014; and accepted 27 August 2014)

\begin{abstract}
This study investigated biomechanical mechanisms of acute subdural hematoma caused by judo and sought preventive measures to reduce injury. A Japanese judo expert repeatedly threw an anthropometric test device using two throwing techniques, Osoto-gari and Ouchi-gari. Linear and angular accelerations of the head were measured. Both throwing techniques resulted in the dummy falling backwards, with the occipital area of the head contacting the mat, and peak linear and angular accelerations being observed when the head contacted the mat. For linear acceleration, the posterior-anterior direction showed the greatest force $(41.0 \pm 2.6 \mathrm{G}$ using Osoto-gari, and $86.5 \pm$ $4.3 \mathrm{G}$ using Ouchi-gari). For angular acceleration, values for sagittal plane rotation were greatest among the three directions measured $\left(3315 \pm 168 \mathrm{rad} / \mathrm{s}^{2}\right.$ using Osoto-gari, and $1328 \pm 201 \mathrm{rad} / \mathrm{s}^{2}$ using Ouchi-gari). We concluded that occipital head contact produced the most forceful longitudinal linear and sagittal plane angular accelerations; subsequent stretches and ruptures of parasagittal bridging veins resulting in acute subdural hematoma. As severe head injuries can result if a person's head comes into contact with the mat, offensive throwing techniques should be restricted to participants able to sufficiently demonstrate the Ukemi technique.
\end{abstract}

Judo is one of the popular sports originated from Japan. Judo is performed at the Olympic Games, and is widely performed in schools and public facilities for education, training, or recreation. Currently, 200 countries are affiliated with the International Judo Federation, with an estimated 20 million participants (10). With the increased popularity of judo comes an increased focus on injuries resulting from the sport. Head injuries, in particular, are frequent in judo athletes. Rates of cerebral concussion in young

Address correspondence to: Prof. Masahito Hitosugi, $\mathrm{MD}, \mathrm{PhD}$

Department of Legal Medicine, Shiga University of Medical Science, Tsukinowa, Otsu, Shiga 520-2192, Japan

Tel: +81-77-548-2200, Fax: +81-77-548-2200

E-mail: hitosugi@belle.shiga-med.ac.jp judo athletes are estimated at 2.38/1000 male athletes and 2.92/1000 female athletes (9). Unfortunately, severe or fatal head injuries during judo have occurred.

The All Japan Judo Federation, in which most instructors and participants at judo halls, schools, and company teams are registered, has started the System for Comparison for Loss or Damage, which records death or severe residual disability. Recently, in Japan, accident reports provided to the All Japan Judo Federation's System for Comparison for Loss or Damage were analyzed (4), and 30 severe head injuries were noted in the last 8 years. In 28 of 30 cases, diagnosis was acute subdural hematoma (ASDH). The common cause of injury was the person being thrown to the mat on their back $(63.3 \%)$, and Osoto-gari was the leading throwing technique 
used resulting in severe head injury.

Judo mats are made from tatami or some similarly acceptable material approved by the International Judo Federation, and most judo training halls are well equipped and the tatami is laid with a shock absorber. Therefore, if someone performing judo hits their head on the mat, marked external injuries are seldom found.

Judo is a martial art using quick throws and it can be difficult to determine exactly how and when a head injury is caused. Therefore, detail mechanisms of the cause of severe head injuries, especially ASDH, during judo are not fully understood, and effective preventive measures have not been implemented.

In Japan, according to revised government teaching guidelines, most junior high school students are required to practice judo from April 2012. This has been a cause of concern to some parents who worry about head injuries resulting from judo. Removing parents' concerns and establishing effective preventive measures are important.

To understand the mechanisms resulting in ASDH from judo, and to assist with creating effective measures to prevent injury, biomechanical analysis was performed.

\section{MATERIALS AND METHODS}

One Japanese male judo expert (26 years old; height,
$177 \mathrm{~cm}$; weight, $90 \mathrm{~kg}$; experience, fifth-dan) repeatedly threw an anthropomorphic test device (ATD). Informed consent was obtained and the study was approved by the Research Ethics Committee of Dokkyo Medical University School of Medicine.

An ATD is a mechanical model of the human body typically used in vehicle crash testing. Using an ATD it is possible to obtain mechanical loading parameters at impact which would be injurious to a person. The POLAR dummy is among a number of state-approved models and was used in the present study (1, 5) (Fig. 1). The POLAR dummy (stature, $175 \mathrm{~cm}$; mass, $75 \mathrm{~kg}$ ) was designed to simulate the kinematics of the human body during car-pedestrian collisions, and has high biofidelity. The close similarity of this dummy to humans has been validated with tests using post-mortem human subjects (5). As people performing judo experience complex rotations or injuries to the body, POLAR is suitable for detecting the physical parameters with high reliability.

On the test day, a tatami (SV230; Hayakawa Textile Industries Co., Ltd., Kashiwara, Osaka, Japan) was laid on a concrete floor. This type of tatami has been used at international judo tournaments. A 6-cm-thick synthetic sponge consisting of urethane and polyethylene (AM2202; Senoh Corp., Matsudo, Chiba, Japan) was placed under the tatami.

Because most ASDH injuries are reported to occur with the Osoto-gari technique, this technique was applied in the current study. In Osoto-gari, the

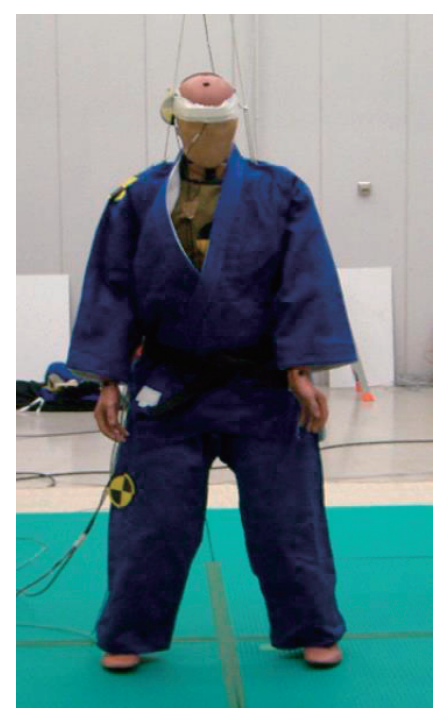

(A)

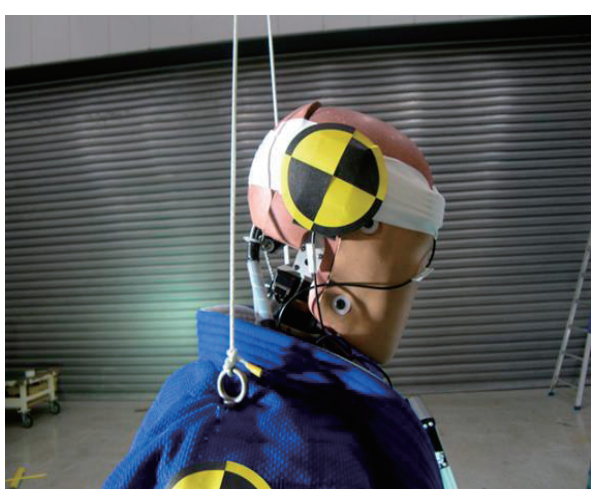

(B)

Fig. 1 Apparatus of the POLAR dummy used in the study (A: full body, B: head). 
thrower breaks the recipient's balance towards the recipient's rear corner and then sweeps the recipient's leg. Subsequently, because of the motion of the body, the recipient is thrown backward. Another similar throwing technique, Ouchi-gari, can also cause ASDH (4), so this technique was also applied in this study. In Ouchi-gari, the thrower pushes the recipient straight back and then sweeps the recipient's leg from the inside with their own leg so that the recipient falls onto their back. The judo expert in the current study threw the ATD eight times using the Osoto-gari $(n=4)$ and Ouchi-gari $(n=4)$ techniques.

Linear acceleration of the head was measured by a tri-axial accelerometer. Head acceleration values were obtained in each direction: ax (longitudinal), ay (lateral), az (vertical). Angular acceleration of the head was measured by three degrees of freedom rotational accelerometers. For angular acceleration, $\alpha \mathrm{x}$ was along the anterior-posterior axis (coronal plane rotation), ay along the medial-lateral axis (sagittal plane rotation), and $\alpha z$ along the superior-inferior axis (horizontal plane rotation) of the head. The accelerometers were mounted at the center of gravity of the ATD's head (Fig. 1). Data were recorded using a high-speed data acquisition system, which could sample at $20 \mathrm{kHz}$, and filtered using a Chan- nel Class 1000 filter. ATD kinematics data were obtained using a high-speed digital video camera recording at 1000 frames per second.

To compare mean acceleration values in each direction, Kruskal-Wallis tests were performed. When a significant difference was observed among the three values, the Steel method, a multiple comparison test for non-parametric data, was performed. A $P$-value $<0.05$ was considered statistically significant.

\section{RESULTS}

When the Osoto-gari and Ouchi-gari techniques were performed, the dummy fell backwards, with the occipital area of the head contacting the mat. Time courses for linear and angular accelerations using Osoto-gari or Ouchi-gari corresponded to the kinematics of the recipient data obtained by video camera. Both peak linear and angular accelerations were observed when the head hit the mat. Representative time courses for linear and angular accelerations in each direction are shown in Fig. 2.

We also examined the peak absolute value for each acceleration measurement for all experiments. For linear acceleration, peak ax had a range from 36.9 to $45.7 \mathrm{G}$, peak ay from 9.9 to $21.7 \mathrm{G}$, and peak

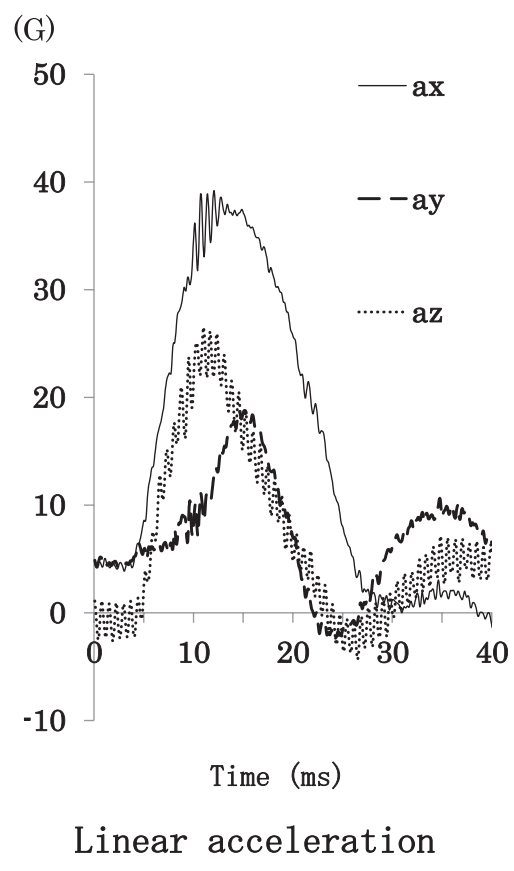

(A)

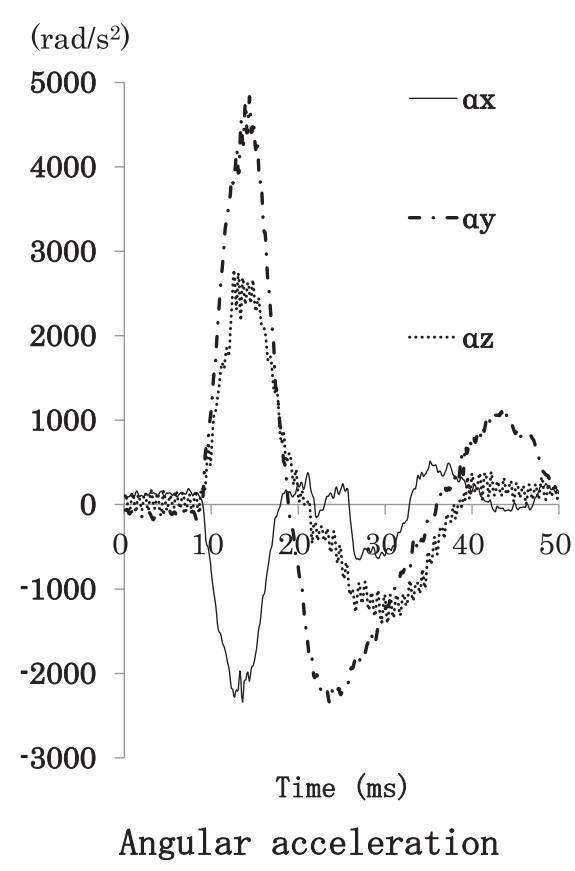

(B)

Fig. 2 Representative time courses for linear (A) and angular (B) accelerations for each direction with Osoto-gari. 


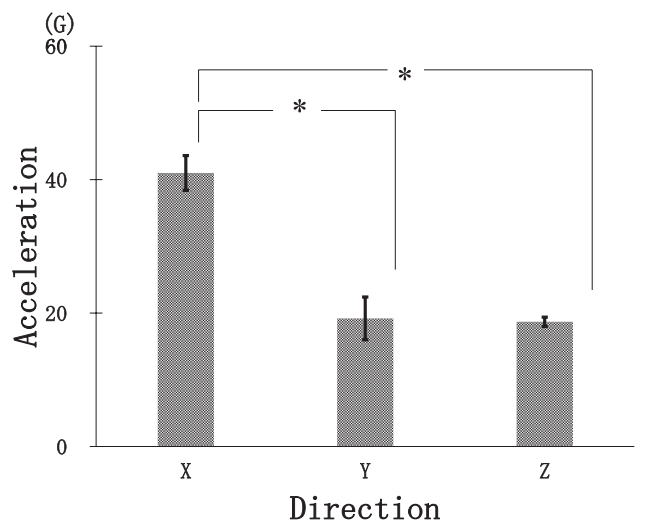

Fig. 3 Comparison of peak absolute values of linear acceleration for each direction with Osoto-gari (statistical significance: $P=0.02$, Kruskal-Wallis test; $P<0.05$, Steel Method).

az from 17.7 to $20.0 \mathrm{G}$ using Osoto-gari. Among the mean \pm standard error (S.E.) values of these three directions $(41.0 \pm 2.6 \mathrm{G}, 19.2 \pm 3.2 \mathrm{G}$, and $18.7 \pm 0.7 \mathrm{G}$, respectively), there was an overall significant difference $(P=0.02$, Kruskal-Wallis test) (Fig. 3). The mean \pm S.E. of the peak ax value was significant greater than those for ay and az $(P<0.05$, Steel method).

Using the Ouchi-gari technique, peak ax had a range from 73.7 to $92.4 \mathrm{G}$, peak ay from 0.8 to $38.3 \mathrm{G}$, and peak az from 10.4 to $22.4 \mathrm{G}$. Among the mean \pm S.E. values of these three directions $(86.5 \pm$ $4.3 \mathrm{G}, 14.5 \pm 8.2 \mathrm{G}$, and $15.1 \pm 2.7 \mathrm{G}$, respectively), there was an overall significant difference $(P=$ 0.001 , Kruskal-Wallis test). The mean \pm S.E. of the peak ax value was significantly greater than those for ay and az $(P<0.05$, Steel method).

For angular acceleration, peak $\alpha \mathrm{x}$ had a range from 965.9 to $1967.5 \mathrm{rad} / \mathrm{s}^{2}$, peak ay from 3044.7 to $3796.3 \mathrm{rad} / \mathrm{s}^{2}$, and peak $\alpha \mathrm{z}$ from 2087.5 to 2530.5 $\mathrm{rad} / \mathrm{s}^{2}$ using Osoto-gari. Among the mean \pm S.E. values of these three directions $\left(1760 \pm 227 \mathrm{rad} / \mathrm{s}^{2}\right.$, $3315 \pm 168 \mathrm{rad} / \mathrm{s}^{2}$, and $2225 \pm 104 \mathrm{rad} / \mathrm{s}^{2}$, respectively), there was an overall significant difference $(P=$ 0.02 , Kruskal-Wallis test) (Fig. 4). The mean \pm S.E. of the peak ay value was significant greater than that of $\alpha \mathrm{x}(P<0.05$, Steel method).

With the Ouchi-gari technique, the peak ax range was from 365.9 to $3072.9 \mathrm{rad} / \mathrm{s}^{2}$, peak ay from 662.5 to $2337.4 \mathrm{rad} / \mathrm{s}^{2}$, and peak $\alpha z$ from 233.8 to $1011.9 \mathrm{rad} / \mathrm{s}^{2}$. Among the mean \pm S.E. values of these three directions $\left(1109 \pm 413 \mathrm{rad} / \mathrm{s}^{2}, 1328 \pm 201\right.$ $\mathrm{rad} / \mathrm{s}^{2}$, and $651 \pm 652 \mathrm{rad} / \mathrm{s}^{2}$, respectively), no overall significant difference was found $(P=0.39$, Kruskal-Wallis test).

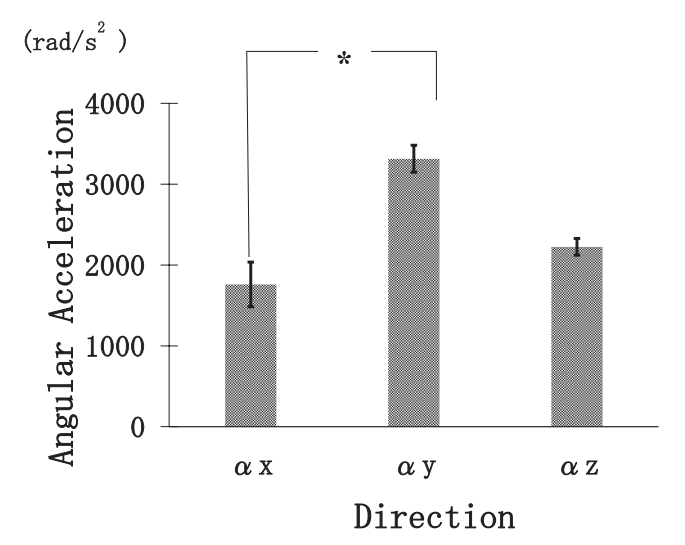

Fig. 4 Comparison of peak absolute values of angular acceleration for each direction with Osoto-gari (statistical significance: $P=0.02$, Kruskal-Wallis test; $P<0.05$, Steel Method).

\section{DISCUSSION}

Because head injuries to young judo participants sometimes lead to life-threatening long-term disability or cognitive impairment it is necessary to have a detailed understanding of the mechanisms causing head injury, and to establish injury prevention strategies. As injury prevention can be improved by educating players and coaches, clarifying the mechanisms are important.

In this study, the mechanism causing severe head injuries, especially ASDH, during judo was able to be reconstructed. We confirmed that when the occipital area of the head struck the mat, high linear acceleration values were obtained in the longitudinal direction. This rapid forceful movement generated a sagittal plane rotational force on the brain. High angular acceleration values were also obtained. Such movement produces stretching and ruptures to parasagittal bridging veins and causes ASDH. Death may also result from the massive increase in intracranial pressure and the resultant marked decrease in cerebral perfusion. Severe head injury can occur when the judo participant fails to perform a falling technique in a manner that minimizes contact of the head with the mat. One technique that minimizes injury is called Ukemi. The Ukemi means that the persons flex their head and neck with their arm extending horizontally when thrown and landing on their back. This technique is required to prevent serious head contact with the ground in judo.

Previously, in most studies on head injuries, linear acceleration has been used to quantify external forces (12). However, it is unlikely that head acceleration in response to a judo throw is isolated to ei- 
ther linear or angular acceleration. In real-world accidents, a high magnitude of angular acceleration requires head contact to occur (8). In our study, high values for both linear and angular accelerations were obtained with head contact. A study examining cerebral concussion from real-world football impact suggested that both linear and angular components of acceleration contributed to concussion, and in about two-thirds of cases, the study subject suffered from front-back impact to the head, indicating sagittal plane rotation of the brain (3). Values obtained in the current study are in accordance with values observed using study subjects suffering from concussion. In other studies, angular acceleration of 6383 $\mathrm{rad} / \mathrm{s}^{2}$ was proposed as the threshold for concussion to football players (11), and a limit of $4500 \mathrm{rad} / \mathrm{s}^{2}$ for concussion was suggested by Ommaya et al. (7). Therefore, the angular acceleration observed in this study (sagittal plane rotation of $3315 \pm 168 \mathrm{rad} / \mathrm{s}^{2}$ using Osoto-gari) may be sufficient to cause ASDH.

As previously suggested, a single biomechanical measure, such as linear acceleration, is not the most sensitive biomechanical measure for determining ASDH risk $(2,6)$. The combination of linear and angular kinematic parameters is likely to have the greatest predictive capabilities for ASDH. For judo, based on reported real-world accidents, reconstructing head impacts, such as those performed in the current study, and accumulating biomechanical parameters may aid in understanding thresholds of accelerations causing ASDH. Further research is required to examine acceleration and head kinematics.

In this study, we obtained high values for angular acceleration, especially with Osoto-gari. This finding is consistent with real-world accident reports that suggest Osoto-gari is the most common technique causing ASDH (4). In Osoto-gari, when the recipient's leg is swept, their body is immediately rotated in a standing position and the occipital area of the head comes into contact with the tatami. Because of the potential to cause injury, this technique should not be used by beginners.

In judo, young beginners or inexperienced people often experience severe head injuries if they do not perform Ukemi correctly, as the occipital area of the head strikes the mat and, subsequently, high values for linear and angular acceleration arise. In research on head injuries suffered in judo, $60 \%$ of participants had been practicing for less than 36 months. Because the major cause of head injuries is a deficiency in the Ukemi technique in beginners, it is recommended that extensive practice of Ukemi is essential for beginners (4).

We confirmed the biomechanical mechanisms causing severe head injuries, in particular ASDH, in judo. Severe contact of the occipital part of the head with the tatami and subsequent high values for both linear and angular accelerations in the sagittal plane could led to the occurrence of ASDH. Therefore, as a primary preventive measure to reduce severe head injuries in judo, youth participants should master Ukemi (the correct way to respond to a fall). Furthermore, as severe head injuries can result if the head of the recipient comes into contact with the mat, throwing techniques should be restricted to participants demonstrating sufficient Ukemi technique.

\section{Acknowledgments}

The authors gratefully acknowledge Honda R\&D Centre in Tochigi for their support. This study was funded in part by a Grant-in-Aid for Young Scientists (A) (No. 17689024) from the Ministry of Education, Culture, Sports, Science and Technology, Japan.

\section{REFERENCES}

1. Akiyama A, Okamoto M and Rangarajan N (2001) Development and application of the new pedestrian dummy. ESV Papers 463, 1-12.

2. Gennarelli TA and Thibault LE (1982) Biomechanics of acute subdural hematoma. J Trauma 22, 680-686.

3. Greenwald RM, Gwin JT, Chu JJ and Crisco JJ (2008) Head impact severity measures for evaluating mild traumatic brain injury risk exposure. Neurosurgery 62, 789-798.

4. Kamitani T, Nimura Y, Nagahiro S, Miyazaki S and Tomatsu $\mathrm{T}$ (2013) Catastrophic head and neck injuries in judo players in Japan from 2003 to 2010. Am J Sports Med 41, 19151921.

5. Kerrigan J, Kore H, Murphy D, Kam C, Bose D and Crandall J (2005) Kinematic corridors for PMHS tested in full-scale pedestrian impact tests. In: Proceedings of the 19th Conference on the Enhanced Safety of Vehicles (ESV) (United States Department of Transportation National Highway Traffic Safety Administration, ed) pp5-394, United States Department of Transportation National Highway Traffic Safety Administration, Washington, DC.

6. Kimpara H and Iwamoto M (2012) Mild traumatic brain injury predictors based on angular accelerations during impacts. Ann Biomed Eng 40, 114-126.

7. Ommaya AK, Goldsmith W and Thibault L (2002) Biomechanics and neuropathology of adult and paediatric head injury. Br J Neurosurg 16, 220-242.

8. Pellman EJ, Viano DC, Tucker AM, Casson IR and Waeckerle JF (2003) Concussion in professional football: reconstruction of game impacts and injuries. Neurosurgery 53, 799-814.

9. Pieter W (2005) Martial arts injuries. In: Epidemiology of Pediatric Sports Injuries: Individual Sports (Medcine and Sport Science), Vol.48 (Caine DJ, Maffuli N, eds) pp59-73, Karger, Basel. 
10. Pocecco E, Ruedl G, Stankovic N, Sterkowicz S, Del Vecchio FB, Gutiérrez-García C, Rousseau R, Wolf M, Kopp M, Miarka B, Menz V, Krüsmann P, Calmet M, Malliaropoulos $\mathrm{N}$ and Burtscher M (2013) Injuries in judo: a systematic literature review including suggestions for prevention. $\mathrm{Br} J$ Sports Med 47, 1139-1143.

11. Rowson S, Duma SM, Beckwith JG, Chu JJ, Greenwald RM,
Crisco JJ, Brolinson PG, Duhaime AC, McAllister TW and Maerlender AC (2012) Rotational head kinematics in football impacts: an injury risk function for concussion. Ann Biomed Eng 40, 1-13.

12. Schmitt K, Niederer P, Muser M and Walz F (2010) Trauma Biomechanics. 3rd Edition. Springer, Heidelberg. 